\title{
Optimal assignment of 4D close-loop instructions to enable CDOs in dense TMAs
}

\author{
Raúl Sáez, Ramon Dalmau and Xavier Prats \\ Department of Physics - Aeronautics Division \\ Technical University of Catalonia - BarcelonaTECH \\ Castelldefels, Spain
}

\begin{abstract}
Continuous descent operations (CDOs) with required times of arrival (RTAs) represent a potential solution to reduce the environmental impact in terminal maneuvering areas without degrading capacity. However, flight management systems require to know the remaining distance to the metering fix in order to compute the CDO complying with the CTA. This paper assesses the feasibility of replacing the current air traffic control sequencing and merging techniques, which are mainly based on open-loop vectoring, by a control based on RTAs over known and pre-defined arrival routes (i.e., with known distances to go). An optimal control problem has been formulated and solved in order to generate CDO trajectories, while a mixedinteger-linear programming model was build in order to solve the aircraft landing problem in the metering fix. The assessment has been performed for Berlin-Schönefeld airport (Germany), by using arrival traffic gathered from historical data and by taking advantage of its tromboning procedure. Furthermore, a second scenario was studied by adding more simulated traffic to the existing one. Results show that, after assigning an RTA and a route to every arriving aircraft, a time separation of 120 is ensured in the metering fix, while at least 90 seconds of separation are maintained in the the rest of waypoints of the procedure.
\end{abstract}

\section{INTRODUCTION}

The reduction of the environmental impact of aviation is one of the major drivers of current research efforts in air transportation. Continuous descent operations (CDOs) enable the execution of a flight profile optimized to the operating capability of the aircraft, giving as a result optimal engineidle descents that reduce fuel consumption, gaseous emissions and noise nuisance [1]-[3]. The International Civil Aviation Organization (ICAO) has published some CDO guidance material [4] to support air navigation service providers (ANSP) to design vertical corridors in which all descent trajectories must be contained, helping in this way to strategically separate them from other procedures in the vicinity. However, as reported in [5], these criteria have been established without explicitly considering the aircraft type, assuming international standard atmosphere (ISA) conditions and with coarse assumptions regarding the aircraft gross mass and performance data. This leads, in the majority of cases, to too restrictive corridors that limit the potential CDO adherence in real operations.

Nonetheless, in busy terminal maneuvering areas (TMAs) this kind of procedures hardly occurs. Instead, air traffic controllers (ATCs) use tactical instructions such as altitude assignments, speed adjustments and radar vectoring so as to maintain safe separation between aircraft and maximize the capacity. However, these techniques tend to degrade the performance of descent operations, leading to a higher environmental impact. In addition, the duration of such "open-loop" vector instructions is not known, nor how the aircraft will re-join its initial route. As a result, it is impossible for state-of-the art flight management systems (FMS) to predict the remaining distance to go and, therefore, to optimize the trajectory to achieve the most environmentally-friendly descent profile.

New air traffic management (ATM) paradigms, as described by SESAR [6], aim to remove this open-loop vectoring by efficiently implementing 4D trajectories. One strategy would be to sequence and merge arrival traffic by assigning required times of arrival (RTAs) at one or several fixes to each aircraft, allowing the FMS to know the remaining distance to go and, thus, enabling the aircraft to fly an optimal descent profile satisfying the RTA [7]. This way, there would be no need for vectoring except for unforeseen situations.

In the recent years, there has been a big effort on the development of advanced concepts and technologies in order to satisfy RTAs with high accuracy [8], [9]. In addition, there are previous works analyzing realistic high-demand scenarios, in which all the descents subject to RTAs are performed with the engines at idle and without speed-brake usage [10]. It has been shown that for low-traffic scenarios the arrival traffic can be managed by only assigning RTAs, while for high traffic loads it would be necessary to notify the RTA well in advance.

In between 4D trajectories with fixed lateral route and openloop vectoring lie some strategies such as point merge (PM) and tromboning. In PM, aircraft fly sequencing legs at a constant altitude, until "direct to" instructions are given to a merge point, used for traffic integration. According to [13], by using PM airlines could save up to $184 \mathrm{~kg}$ of fuel per aircraft and the mean controller task load and the number of instructions to pilots could be reduced by a $20 \%$ and a $30 \%$ respectively. Very promising results can be also found in [12], with even better results regarding the controllers workload. However, the environmental benefits of the PM are limited to altitudes below the sequencing leg altitude, since the remaining distance is known with certainty only after the "direct to" instruction. Even if the CDO were initiated at the cruise level, it would be interrupted to maintain level flight while following the sequencing leg before cleared to the merge point, leading to an increase in fuel burnt while leveling off at relatively low altitudes. The tromboning is a trombone- 
shape RNAV procedure consisting in a set of parallel legs composed of multiple waypoints, in which ATC may give a shortcut (depending on the traffic) to the next leg reducing the total descent length. Previous works [11] proposed a concept consisting of separating, sequencing and merging traffic by negotiating RTAs and shortcuts between aircraft and ATC before starting the descent. Results from a preliminary study show that, for a given RTA, several shortcuts could be assigned such that the RTA fits into the feasible time window.

To the best of the author's knowledge, no other works analyzed a high-demand scenario with real traffic, in which aircraft fly CDO trajectories from cruise level to the runway threshold by following a real trombone procedure. The solution proposed consists in managing high traffic scenarios without using open-loop vectors by combining not only the assignment of RTAs to every arriving aircraft, but also the assignment of shortcuts, ensuring separation between aircraft in the trombone procedure. The assessment has been performed for Berlin-Schönefeld airport (Germany) by using arrival traffic gathered from historical data during a busy day. A second scenario with additional traffic has been also studied.

\section{BACKGROUND}

In this paper, the earliest and latest descent trajectories and the trajectory that minimizes the operating cost were computed for each aircraft arriving at the airport subject of study and for each possible alternative shortcut of the tromboning procedure.

Given the feasible time window and the target time of arrival of each flight in the landing sequence, the aircraft landing problem was solved to ensure safe separation at the different waypoints of the tromboning procedure while minimizing deviations from the preferred times of arrival.

Section II-A presents a method to generate the optimal trajectories; Section II-B describes the aircraft landing problem.

\section{A. Trajectory optimisation problem}

Given a known lateral route (shortcut), and consequently a fixed distance to go, the optimization of the vertical profile (altitude and speed) can be formulated as an optimal control problem, which aims at computing the control time history of a system, here the aircraft, such that a cost function is minimized while satisfying some dynamic and operational constraints.

1) Generic optimal control problem: A generic optimal control problem is defined as follows:

$$
\begin{array}{ll}
\min _{\boldsymbol{u}(t)} & J_{\text {ocp }}:=\phi\left(\boldsymbol{x}\left(t_{f}\right)\right)+\int_{t_{0}}^{t_{f}} L(\boldsymbol{x}(t), \boldsymbol{u}(t), \boldsymbol{p}) \mathrm{d} t \\
\mathrm{s.t} & \boldsymbol{x}\left(t_{0}\right)=\boldsymbol{x}_{0} \\
& \dot{\boldsymbol{x}}(t)=\boldsymbol{f}(\boldsymbol{x}(t), \boldsymbol{u}(t), \boldsymbol{p}) \\
& \boldsymbol{h}(\boldsymbol{x}(t), \boldsymbol{u}(t), \boldsymbol{p}) \leq 0 \\
& \boldsymbol{\psi}\left(\boldsymbol{x}\left(t_{f}\right)\right)=0
\end{array}
$$

where $\boldsymbol{x} \in \mathbb{R}^{n_{x}}$ is the state vector, with fixed initial conditions $\boldsymbol{x}_{0} ; \boldsymbol{u} \in \mathbb{R}^{n_{u}}$ is the control vector; and the vector $\boldsymbol{p} \in \mathbb{R}^{n_{p}}$ includes all the time-independent parameters of the model; $L: \mathbb{R}^{n_{x}} \times \mathbb{R}^{n_{u}} \times \mathbb{R}^{n_{p}} \rightarrow \mathbb{R}$ and $\phi: \mathbb{R}^{n_{x}} \rightarrow \mathbb{R}$ are the
Lagrange and Mayer terms of the cost function, respectively. The dynamics of the state vector are expressed by a set of non-linear equations $\boldsymbol{f}: \mathbb{R}^{n_{x}} \times \mathbb{R}^{n_{u}} \times \mathbb{R}^{n_{p}} \rightarrow \mathbb{R}^{n_{x}} ; \boldsymbol{h}: \mathbb{R}^{n_{x}} \times$ $\mathbb{R}^{n_{u}} \times \mathbb{R}^{n_{p}} \rightarrow \mathbb{R}^{n_{h}}$ and $\boldsymbol{\psi}: \mathbb{R}^{n_{x}} \rightarrow \mathbb{R}^{n_{\psi}}$ represent applicable path and terminal constraints, respectively. Note that if the time interval is not fixed, $t_{f}$ becomes a new decision variable.

The Hamiltonian of the optimal control problem (1) is:

$$
H=L+\boldsymbol{\lambda}^{T} \boldsymbol{f}+\boldsymbol{\mu}^{T} \boldsymbol{h}
$$

where $\boldsymbol{\lambda}$ and $\boldsymbol{\mu}$ are vectors of Lagrange multipliers. The set of necessary conditions for $J$ to be stationary optimum is [14]:

$$
\begin{aligned}
\dot{\boldsymbol{\lambda}} & =-\left(\frac{\partial H}{\partial \boldsymbol{x}}\right) \\
0 & =\left(\frac{\partial H}{\partial \boldsymbol{u}}\right) \\
\boldsymbol{\lambda}\left(t_{f}\right) & =\left(\frac{\partial \phi}{\partial \boldsymbol{x}}+\boldsymbol{\nu}^{T} \frac{\partial \boldsymbol{\psi}}{\partial \boldsymbol{x}}\right)_{t=t_{f}}^{T} \\
0 & =\left[\left(\begin{array}{ll}
\frac{\partial \phi}{\partial \boldsymbol{x}}+\boldsymbol{\nu}^{T} \frac{\partial \boldsymbol{\psi}}{\partial \boldsymbol{x}} & f+L
\end{array}\right]_{t=t_{f}}\right. \\
\boldsymbol{\mu} & =\left\{\begin{array}{l}
\geq 0 \text { if } \boldsymbol{h}=0 \\
=0 \text { if } \boldsymbol{h}<0
\end{array}\right.
\end{aligned}
$$

2) Optimal control problem for aircraft descents: The state vector $\boldsymbol{x}=[v, h, s]$ is composed of the true airspeed (TAS), the altitude of the aircraft, and the distance to go. In order to obtain environmentally friendly trajectories, idle thrust is assumed and speed brakes use is not allowed throughout the descent. In such conditions, the flight path angle is the only control (i.e., $\boldsymbol{u}=[\gamma]$ ), which is used to manage the energy of the aircraft and achieve different times of arrival at the metering fix with minimum fuel consumption and noise nuisance.

The dynamics of $\boldsymbol{x}$ are expressed by the following set of ordinary differential equations (ODE), considering a point-mass representation of the aircraft reduced to a "gamma-command" model, where vertical equilibrium is assumed (lift balances weight). In addition, the cross and vertical components of the wind are neglected, and the aerodynamic flight path angle is assumed to be small (i.e., $\sin \gamma \simeq \gamma$ and $\cos \gamma \simeq 1$ ):

$$
\boldsymbol{f}=\left[\begin{array}{c}
\dot{v} \\
\dot{h} \\
\dot{s}
\end{array}\right]=\left[\begin{array}{c}
\frac{T_{i d l e}-D}{m}-g \gamma \\
v \gamma \\
v+w
\end{array}\right]
$$

where $T_{\text {idle }}: \mathbb{R}^{n_{x}} \rightarrow \mathbb{R}$ is the idle thrust; $D: \mathbb{R}^{n_{x} \times n_{u}} \rightarrow \mathbb{R}$ is the aerodynamic drag; $g$ is the gravity acceleration and $m$ the mass, of which is assumed to be constant because the fuel consumption during an idle descent is a small fraction of the total $m$ [3]. The longitudinal component of the wind $w: \mathbb{R} \rightarrow \mathbb{R}$ is modelled by a smoothing spline [15]:

$$
w(h)=\sum_{i=1}^{n_{c}} c_{i} B_{i}(h)
$$

$B_{i}, i=1, \ldots, n_{c}$, are the $\mathrm{B}$-spline basis functions and $\boldsymbol{c}=\left[c_{1}, \ldots, c_{n_{c}}\right]$ are control points of the smoothing spline. It 
should be noted that the longitudinal wind has been modelled as a function of the altitude only, as done in similar works [16]. The control points of the spline approximating the longitudinal wind profile are obtained by fitting historical weather data.

In this paper, the trajectory is divided in two phases: the latter part of the cruise phase prior the top of descent (TOD), and the idle descent down to the metering fix where the RTA will be assigned. Assuming that the original cruise speed will not be modified after the optimization process, the twophases optimal control problem can be converted into a singlephase optimal control problem with the following Lagrange term [17], which takes into account both fuel and time costs:

$$
L=-\left.\left(\frac{f+\mathrm{CI}}{v+w}\right)\right|_{\boldsymbol{x}=\boldsymbol{x}_{\mathbf{0}}}(v+w)+f_{i d l e}+\mathrm{CI}
$$

where $f: \mathbb{R}^{n_{x} \times n_{u}} \rightarrow \mathbb{R}$ and $f_{\text {idle }}: \mathbb{R}^{n_{x}} \rightarrow \mathbb{R}$ are the nominal and idle fuel flow, respectively; and CI is the cost index ${ }^{1}$.

In addition to the dynamic constraints $f$, the following set of path constraints are enforced to ensure that the aircraft airspeed remains within operational limits, and that the maximum and minimum descent gradients are not exceeded:

$$
\boldsymbol{h}=\left[\begin{array}{c}
v_{C A S, \min }-v_{C A S} \\
v_{C A S}-\mathrm{VMO} \\
M-\mathrm{MMO} \\
\gamma \\
\gamma_{\min }-\gamma
\end{array}\right] \leq\left[\begin{array}{l}
0 \\
0 \\
0 \\
0 \\
0
\end{array}\right]
$$

where $v_{C A S}: \mathbb{R}^{n_{x}} \rightarrow \mathbb{R}$ is the calibrated airspeed (CAS) and $M: \mathbb{R}^{n_{x}} \rightarrow \mathbb{R}$ is the Mach number, both functions of the state vector; $v_{C A S, \text { min }}$ and VMO are the minimum and maximum operative CAS, respectively; MMO is maximum operative Mach; and $\gamma_{\min }$ is the minimum descent gradient.

Different alternatives can be used to model the aircraft performance functions $T_{\text {idle }}, D, f$ and $f_{\text {idle }}$ and their respective parameters. In this paper, the EUROCONTROL's base of aircraft data (BADA) v4 model has been adopted [18].

Finally, terminal constraints fix the final states vector:

$$
\boldsymbol{\psi}=\left[\begin{array}{c}
v-v_{R T A} \\
h-h_{R T A} \\
s-s_{R T A}
\end{array}\right]=\left[\begin{array}{l}
0 \\
0 \\
0
\end{array}\right]
$$

where $\boldsymbol{x}_{R T A}=\left[v_{R T A}, h_{R T A}, s_{R T A}\right]$ is the state vector at the metering fix where the RTA will be assigned by ATC.

In the formulation presented herein, there is only one control variable, which appears linearly in the equations describing the dynamics of the system as well in the cost function to be minimized. Consequently, the Hamiltonian of the system (2) is also linear with respect to the control, leading to a singular optimal control problem which can be solved semi-analytically from the implicit formulation of optimal singular arcs [17].

Since the initial and final states of the trajectory are fixed, the optimal trajectory will be of a "bang-singular-bang" type. These solutions are composed by three arcs: one initial bang arc with the control variable at its maximum or minimum value

\footnotetext{
${ }^{1}$ The cost index is a parameter chosen by the airspace user that reflects the relative importance of the cost of time with respect to fuel costs
}

to go from $x_{0}$ to the singular arc; a singular arc where the optimal control is given as a function of the sates vector; and a final bang arc to go from the singular arc to the final state.

The analytical expression of the optimal control in the singular arc for the above model, and the steps to generate an optimal trajectory semi-analytically can be found in [17].

As an example, Fig. 1 shows the optimal speed profile for a Boeing B738, in international standard atmospheric (ISA) conditions and no wind. The cost index used to compute the optimal trajectory was $57 \mathrm{~kg} \mathrm{~min}^{-1}$. It can be observed how the optimal speed profile lies in between the boundaries delimited by $v_{C A S, \min }$ and MMO/VMO. Also note that since these maximum and minimum speeds are given in terms of CAS and Mach, the corresponding TAS changes with altitude. The earliest and latest trajectories would correspond to the $v_{C A S, \min }$ and MMO/VMO speed profiles, respectively.

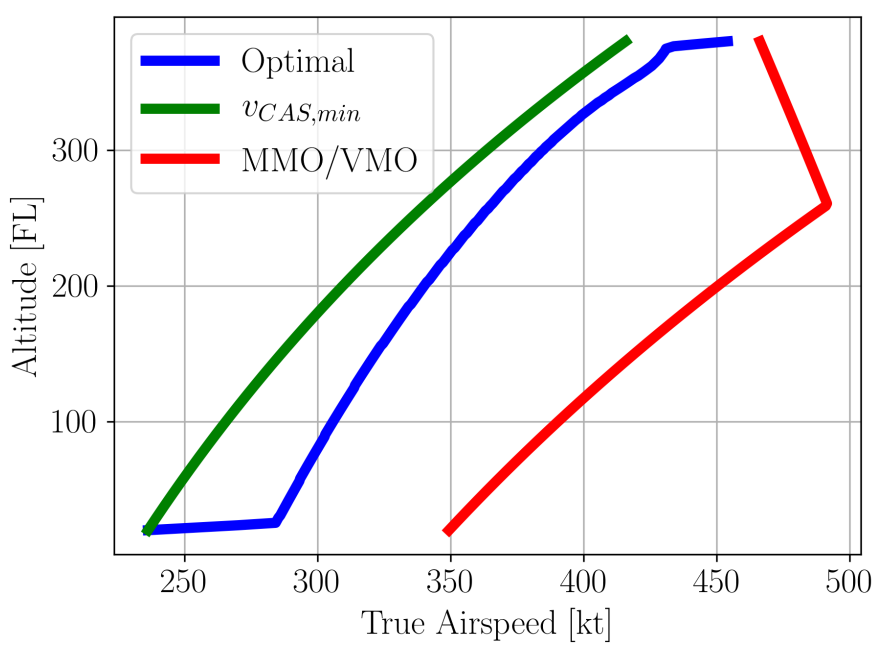

Fig. 1. Example of optimal trajectory

\section{B. Aircraft landing problem}

The set $\mathcal{A}$ is composed by all aircraft scheduled to land at a given airport during a certain period of time, and $\mathcal{P}$ contains all possible pairs of aircraft $(p, q)$, with $p, q \in \mathcal{A}$ and $p \neq q$.

It is assumed that well before the TOD, each aircraft $p$ has computed the optimal descent trajectory according to its specific CI by solving the optimal control problem presented in the previous section. From this trajectory, the expected time of arrival at a certain metering fix, $\mathrm{STA}^{p}$, can be determined.

In addition, the earliest and latest times of arrival at the metering fix $\left(\mathrm{ETA}^{p}\right.$ and $\mathrm{LTA}^{p}$, respectively) are assumed to be known. The earliest and latest trajectories at the metering fix can be computed by solving the optimal control problem with very large positive and negative CI values, respectively.

The goal of the aircraft landing problem is to schedule the arrival traffic by assigning RTAs at a metering fix to each aircraft. Each RTA must fit within the corresponding feasible time window and must ensure separation $t_{s e p}$ between aircraft.

The solution of this problem could be feasible or infeasible depending on the complexity of the scenario. For those feasible 
scenarios, there may exist many distinct solutions such that all the constraints of the problem are satisfied; namely, time separation between aircraft is ensured and the RTA assigned to each aircraft fits within its feasible time window. In such case, the RTA assigned to each aircraft should be as close as possible to the corresponding expected time of arrival in order to minimize the impact of the RTA on the cost of the operation. This optimization problem can be stated as follows:

$$
\begin{array}{ll}
\min _{\substack{\mathrm{RTA}_{p \in \mathcal{A}}^{p} \\
p \in{ }^{2}}} & J_{l d g}:=\sum_{p \in \mathcal{A}}\left|\mathrm{RTA}^{p}-\mathrm{STA}^{p}\right| \\
\text { s.t } & \mathrm{ETA}^{p} \leqslant \mathrm{RTA}^{p} \leqslant \mathrm{LTA}^{p}, \forall p \in \mathcal{A} \\
& \left|\mathrm{RTA}^{p}-\mathrm{RTA}^{q}\right| \geqslant t_{\text {sep }}, \forall(p, q) \in \mathcal{P}
\end{array}
$$

Problem (9) is a discontinuous non-linear programming (DNLP) optimization problem because of the absolute value functions, which lead to discontinuous derivatives. Fortunately, the problem can be easily linearized through a proper reformulation of the absolute value expressions appearing in the cost function and constraints. The resulting linear problem is easier to handle from a numerical point of view.

On the one hand, the separation constraint can be reformulated by means of the following disjunctive constraints:

$$
\begin{aligned}
& \text { RTA }^{p}+t_{\text {sep }} \leq \mathrm{RTA}^{q}+M^{p, q} B^{q, p}, \forall(p, q) \in \mathcal{P}, q<p \\
& \text { RTA }^{p}+t_{\text {sep }} \leq \mathrm{RTA}^{q}+M^{p, q} C^{p, q}, \forall(p, q) \in \mathcal{P}, p<q,
\end{aligned}
$$

where $B^{p, q}$ for $p<q$ is a binary variable that takes a value of 1 if aircraft $p$ arrives before aircraft $q$ and 0 otherwise, $C^{p, q}=1-B^{p, q}$ and $M^{p, q}$ is a relatively large scalar [19]. To avoid problems with numerical stability, the value of $M^{p, q}$ should not be chosen too large. For this particular problem, the minimum $M^{p, q}$ yet high enough to satisfy Eq. (10), can be derived as follows:

$$
M^{p, q}=\max \left\{\mathrm{LTA}^{p}-\mathrm{ETA}^{q}, \operatorname{LTA}^{q}-\mathrm{ETA}^{p}\right\}, \forall(p, q) \in \mathcal{P} .
$$

On the other hand, a common approach to linearize the cost function consists on expressing the term inside the absolute value as the difference of two artificial and positive variables [20], namely $t_{+}^{p}$ and $t_{-}^{p}$, and re-writing the absolute value function in Eq. (6) as the sum of these two variables:

$$
\left|\mathrm{RTA}^{p}-\mathrm{STA}^{p}\right|=t_{+}^{p}+t_{-}^{p}, \forall p \in \mathcal{A} .
$$

By combining Eqs. (10)-(12), the DNLP aircraft landing problem (9) can be re-formulated as the following mixedinteger linear programming (MILP) problem, in which some of the variables are restricted to be integers and the objective function and the constraints are linear:

$$
\begin{array}{ll}
\min _{\substack{\mathrm{RTA}_{p \in \mathcal{A}}^{p} \\
p \in \mathcal{A}}} & J_{l d g}^{*}:=\sum_{p \in \mathcal{A}} t_{+}^{p}+t_{-}^{p} \\
\text { s.t } & \mathrm{ETA}^{p} \leqslant \mathrm{RTA}^{p} \leqslant \mathrm{LTA}^{p}, \forall p \in \mathcal{A} \\
& \mathrm{RTA}^{p}+t_{\text {sep }} \leq \mathrm{RTA}^{q}+M^{p, q} B^{q, p}, \forall(p, q) \in \mathcal{P}, q<p \\
& \operatorname{RTA}^{p}+t_{\text {sep }} \leq \mathrm{RTA}^{q}+M^{p, q} C^{p, q}, \forall(p, q) \in \mathcal{P}, p<q \\
& B^{p, q} \in\{0,1\}, \forall(p, q) \in \mathcal{P}, p<q \\
& \mathrm{RTA}^{p}-\mathrm{STA}^{p}=t_{+}^{p}-t_{-}^{p}, \forall p \in \mathcal{A} \\
& t_{+}^{p}, t_{-}^{p} \geq 0, \forall p \in \mathcal{A}
\end{array}
$$

This formulation of the aircraft landing problem assumes that the time windows for all the pairs of aircraft could overlap. However, in practical scenarios this assumption is not efficient, since there may exist redundant variables and constraints unnecessary increasing the complexity of the problem.

A more efficient algorithm can be developed by classifying the pairs of aircraft into three sets [19]: The first set $\mathcal{P}_{\text {sep }}$ contains pairs of aircraft $(p, q)$ with guaranteed separation, i.e. where $\mathrm{LTA}^{p}+t_{\text {sep }} \leqslant \mathrm{ETA}^{q}$ and $\mathrm{LTA}^{q}+t_{\text {sep }} \leqslant \mathrm{ETA}^{p}$ is fulfilled. For these pairs of aircraft separation is inherently satisfied regardless of the assigned RTAs. The second set $\mathcal{P}_{\text {dis }}$ is composed by the pairs of aircraft $(p, q)$ with disjoint time windows without guaranteed separation, i.e. where $\mathrm{LTA}^{p} \leqslant$ $\mathrm{ETA}^{q}$ and $\mathrm{LTA}^{q} \leqslant \mathrm{ETA}^{p}$ but $\mathrm{LTA}^{p}+t_{\text {sep }}>\mathrm{ETA}^{q}$ or $\mathrm{LTA}^{q}+$ $t_{\text {sep }}>\mathrm{ETA}^{p}$ applies. The last set $\mathcal{P}_{\text {ovlp }}$ contains the pairs of aircraft $(p, q)$ with overlapping time windows. Considering these sets, the following constraints can be added to Eq. (13) aiming to reduce the computational burden:

$$
\begin{aligned}
& \forall(p, q) \in \mathcal{P}_{\text {sep }} \cup \mathcal{P}_{d i s}, \mathrm{LTA}^{p}<\mathrm{ETA}^{q}: B^{p, q}=1 \\
& \forall(p, q) \in \mathcal{P}_{\text {sep }} \cup \mathcal{P}_{d i s}, \mathrm{LTA}^{q}<\mathrm{ETA}^{p}: B^{q, p}=1 \\
& \forall(p, q) \in \mathcal{P}_{d i s}, \mathrm{LTA}^{p}<\mathrm{ETA}^{q}: \mathrm{RTA}^{p}+t_{\text {sep }} \leq \mathrm{RTA}^{q} \\
& \forall(p, q) \in \mathcal{P}_{d i s}, \mathrm{LTA}^{q}<\mathrm{ETA}^{p}: \mathrm{RTA}^{q}+t_{\text {sep }} \leq \mathrm{RTA}^{p}
\end{aligned}
$$

Constraints defined by Eqs. (14a) and (14b) enforce the variables $B^{p, q}$ to be either 0 or 1 in the case of disjoint time windows. The constraints defined by Eqs. (14c) and (14d) are simple precedence constraints.

\section{RESULTS}

This section presents the experimental setup and results obtained after the assessment made for the tromboning arrival procedure of Berlin-Schönefeld airport.

\section{A. Experimental setup}

1) Procedure: In order to merge and sequence aircraft in dense traffic situations, trombone transitions to final approach have been implemented in several airports, being one of them Berlin-Schönefeld, the airport chosen for this study. Trombone procedures are similar to typical vectoring patterns, yet path shortening is accomplished by assigning a finite set of routes, also known as shortcuts, at equally spaced waypoints.

Berlin-Schönefeld has one runway (07L/25R), which is used both for arrivals and departures. In this paper, the arrival and approach procedures for runway 07L were used. More specifically, the north and south standard arrival route (STAR) 


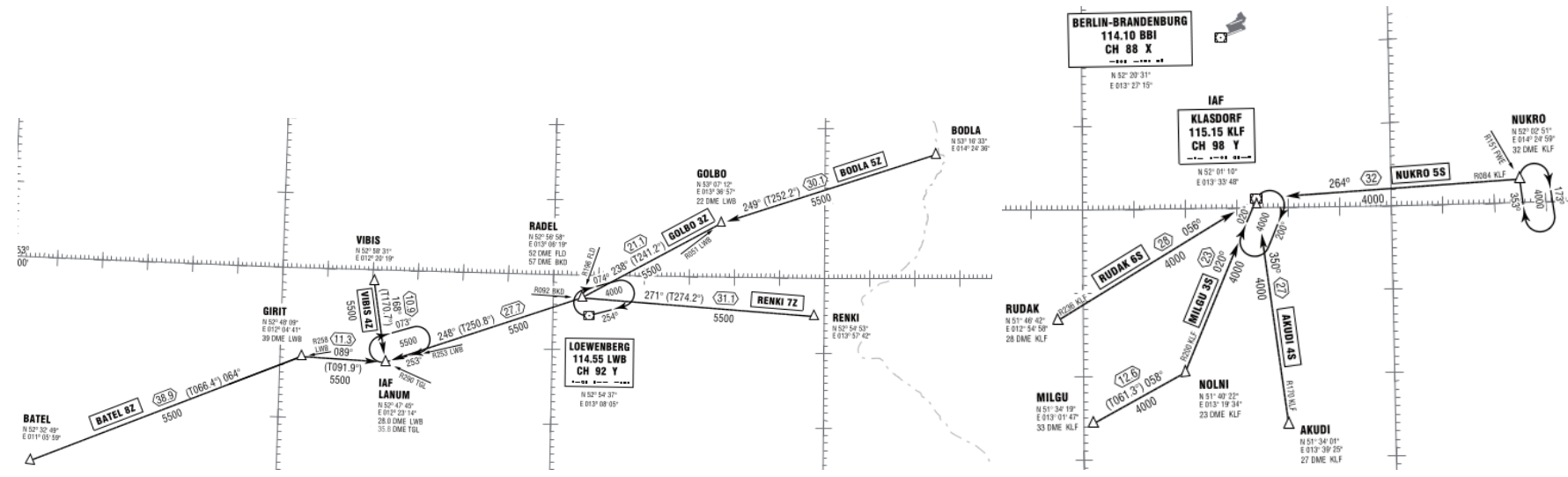

(a) STAR (North) RWY 07L (source: German AIP)

(b) STAR (South) RWY 07L (source: German AIP)

Fig. 2. Berlin-Schönefeld STARs RWY 07L (source: German AIP)

procedures for runway 07L (see Fig. 2), and the GPS/FMS RNAV 07L transition to final approach (see Fig. 3).

Regarding the trombone procedure shown in Fig. 3, it can be observed that it starts in LANUM and KLASDORF, the two initial approach fixes (IAFs). Then, arriving aircraft proceed to the trombone-shaped path, which starts at DB531 and DB541 respectively. From this point on, the ATC could eventually give a shortcut at any of the regularly spaced waypoints, depending on the volume of traffic. For each STAR, there are only 5 possible routes, as it is only possible to assign 5 shortcuts. For the north arrivals, the shortcuts are given in waypoints from DB532 to DB536 (both included), and for the south arrivals in waypoints from DB542 to DB546 (both included). Table I shows the potential routes for the north STAR. For instance, in the route 04 a shortcut is given at DB533 towards DB553, skipping the waypoints from DB534 to DB554 and reducing by $24 \mathrm{NM}$ the total distance flown.

An hypothetical extended TMA (E-TMA) of $250 \mathrm{NM}$ of radius was defined around the airport. As soon as aircraft entered this area, they were assigned with a RTA and a route. Then, it was assumed that they flew straight to the beginning of the STAR, after which they started the tromboning procedure.

TABLE I

POTENTIAL ROUTES FOR BERLIN TROMBONE PROCEDURE (NORTH CASE)

\begin{tabular}{ccc}
\hline ID & Waypoints sequence & Distance [NM] \\
\hline 01 & LANUM-...-DB531-...-DB536-DB556-...-PIKOV & 96.2 \\
02 & LANUM-..-DB531-..-DB535-DB555-...-PIKOV & 88.2 \\
03 & LANUM-..-DB531-..-DB534-DB554-...-PIKOV & 80.2 \\
04 & LANUM-..-DB531-..-DB533-DB553-...-PIKOV & 72.2 \\
05 & LANUM-...-DB531-DB532-DB552-PIKOV & 64.2 \\
\hline
\end{tabular}

2) Input data: The assessment was performed by using flight traffic data from the $10^{\text {th }}$ August 2017. These data were obtained from EUROCONTROL's data demand repository (DDR2) [21], which contains information about the trajectories flown every day. In that day, there were 143 arriving flights.

As Berlin-Schönefeld is not one of the busiest airports in Europe, a second traffic scenario was generated in order to

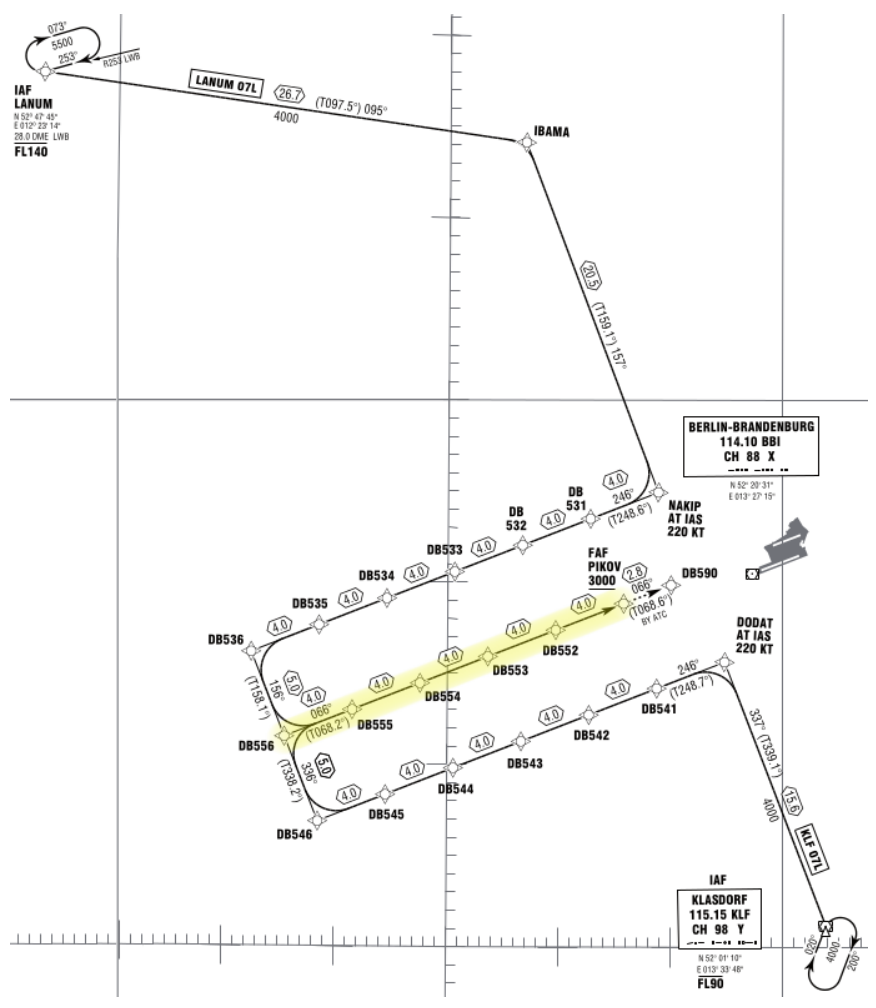

Fig. 3. Berlin-Schönefeld GPS/FMS RNAV 07L Tromboning (source: German AIP)

assess the performance of the proposed approach in a denser traffic situation. It was observed that during the analyzed day, the Airbus A320 and other aircraft comparable in terms of mass, dimensions and performance (e.g., a Boeing 737) represented the $88 \%$ of all arriving aircraft. Therefore, Airbus A320 was selected as the representative aircraft for this study.

The traffic time distribution from the baseline scenario (the one with real traffic) was taken as a reference in order to generate additional traffic. For instance, more traffic is generated at $10 \mathrm{am}$ than at $11 \mathrm{pm}$. In the end, the scenario with additional traffic represented double the traffic of that of 
the baseline scenario, 286 flights. Furthermore, a time shift of a few minutes in the time of entry to the E-TMA of some aircraft was necessary in order to obtain a feasible solution of the aircraft landing problem. In "real-life" operations, this time shift is supposed to be achieved during the en-route phase.

From now on, and for the sake of simplicity, the baseline scenario will be referred as scenario 1 and the scenario with additional traffic will be referred as scenario 2 .

For the optimization of CDO trajectories, the upper bounds on the speed limits (VMO and MMO), which are aircraft dependent, were obtained from the BADA v4 aircraft performance files; the minimum speeds $v_{C A S, \text { min }}$ corresponded to the green dot speed $^{2}$; and the minimum descent gradient was set to $-7^{\circ}$. The weather data to generate the longitudinal wind profile as a function of the altitude were obtained from gridded binary (GRIB) formated files provided by the global forecast system (GFS) of the National Oceanic and Atmospheric Administration (NOAA). For each arriving flight, the GRIB file corresponding to its entry at the E-TMA time was used. Finally, the CI of each flight was inferred from the DDR2 data, following the methodology explained below.

For the aircraft landing problem, the time separation $t_{s e p}$ ensured in the final approach fix (FAF) is, at least, $120 \mathrm{~s}$. The $t_{\text {sep }}$ in the rest of the trombone procedure is, at least, $90 \mathrm{~s}$.

3) Estimation of cost index: As described in Section II-A, the estimated time of arrival for each aircraft depends on the cost index. Unfortunately, this parameter is not shared by airlines because it gives insights on their market strategy.

In this paper, the CI of each flight is estimated from the trajectory as reported in the DDR2, the BADA v4 performance model and historical weather data obtained from GFS GRIBs.

The CI is estimated by assuming that the aircraft was flying at the optimal speed in the cruise phase. In practice, the optimal cruise Mach $\left(M_{\text {opt }}\right)$ is computed by the FMS as a function of the longitudinal wind, temperature $(\tau)$ and pressure $(p)$ at the cruise altitude, the aircraft mass and the CI. The goal is to minimize the following cost function in a given range:

$$
J_{c r z}:=\int_{s_{0}}^{s_{f}} \frac{\mathrm{CI}+f(m, \tau, p, M)}{v(M, \tau)+w} \mathrm{~d} s
$$

Under certain assumptions [22], the variational calculus Euler-Lagrange theorem allows the reduction of the integral optimization problem into a point root-finding problem:

$$
\frac{\partial g}{\partial M}(\mathrm{CI}, m, \tau, p, M, w)=0 \rightarrow M_{o p t}(\mathrm{CI}, m, \tau, p, w)
$$

where $g$ is a certain function that depends on the flight conditions, cost index and aircraft performance [22].

The inverse procedure can be performed to estimate the $\mathrm{CI}$ when $M_{o p t}$ and all the other variables are known:

$$
\frac{\partial g}{\partial M}(\mathrm{CI}, m, \tau, p, M, w)=0 \rightarrow \hat{\mathrm{CI}}\left(M_{o p t}, m, \tau, p, w\right)
$$

\footnotetext{
${ }^{2}$ For the Airbus A320, the green dot speed is the minimum operating speed in managed mode and clean configuration, being approximately the best liftto-drag ratio speed.
}

$M_{o p t}$ is estimated from the segments of the trajectory at constant altitude before the TOD; the weather data are obtained from the GRIB file for the corresponding date and geographical location; and the mass is assumed to be that corresponding to $90 \%$ of the maximum landing mass (MLM).

4) Route assignment: Once the RTAs for every aircraft have been assigned in the FAF by solving the aircraft landing problem, the next step is to assign the routes (i.e., tromboning shortcuts). In the final segment of the trombone procedure (highlighted in yellow in Fig. 3) north and south fluxes are merged, and there is where conflicts may appear. In order to perform the route assignment, time separation between aircraft must be ensured in this final segment between the two arriving fluxes. An iterative process, in which aircraft are assigned always the shortest possible route, is needed in order to avoid potential conflicts. The steps performed by the route assigner algorithm are described below:

1) Check the arriving aircraft sequence in the FAF after assigning the RTAs with the aircraft landing problem.

2) For each aircraft, find which routes enable the attainment of the assigned RTA. The idea is to check whether the RTA is within the time window of each route or not.

3) From the available routes, start always by trying to assign the shortest route.

4) If by assigning the shortest route the time separation in the tromboning final segment is not ensured, try to assign the next shortest route and so on.

In the end, all aircraft will be assigned the shortest possible route, which will allow them to attain the RTA assigned at the FAF during the aircraft scheduling process without incurring a time separation loss between the two inbound fluxes.

\section{B. Earliest and latest trajectories}

Figure 4 shows the earliest and latest trajectories for one of the flights arriving to Berlin Schönefeld. More specifically, they correspond to a B738 flying the AKUDI STAR and the route 02 of the tromboning procedure (see Table I).

It is important to remark that, as explained in Section II-A, the CDO trajectory optimizer used in this study assumes constant cruise speed. As a result, it is not possible to observe any speed change during the cruise phase in Fig. 4. However, after the top of descent it can be noticed that, in the earliest case, there is an acceleration to MMO aiming to arrive as fast as possible, followed by an exchange of potential and kinetic energy by means of elevator control, aiming to achieve VMO. On the other hand, the latest trajectory slows down to $v_{C A S, \min }$ immediately in order to release time.

The computation of these trajectories is of high importance for the work presented in this paper, as it allows to know the earliest and latest times of arrival to the FAF and, therefore, the feasible time window for each distance to go corresponding to a tromboning shortcut. These time windows are used as limiting bounds during the aircraft landing problem formulation. 


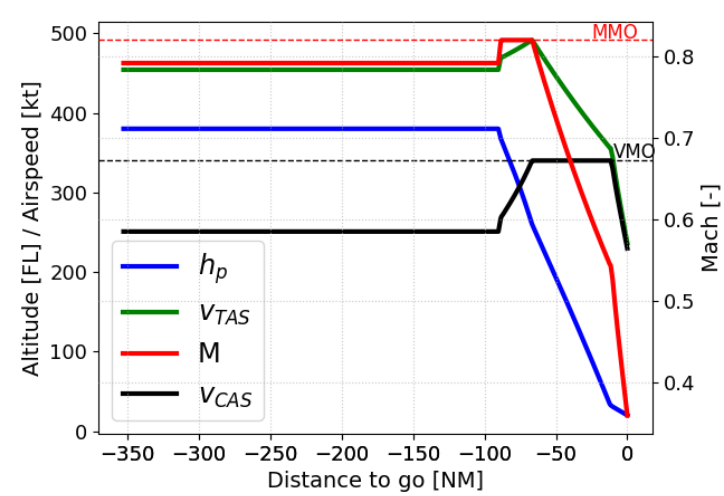

(a) Earliest trajectory

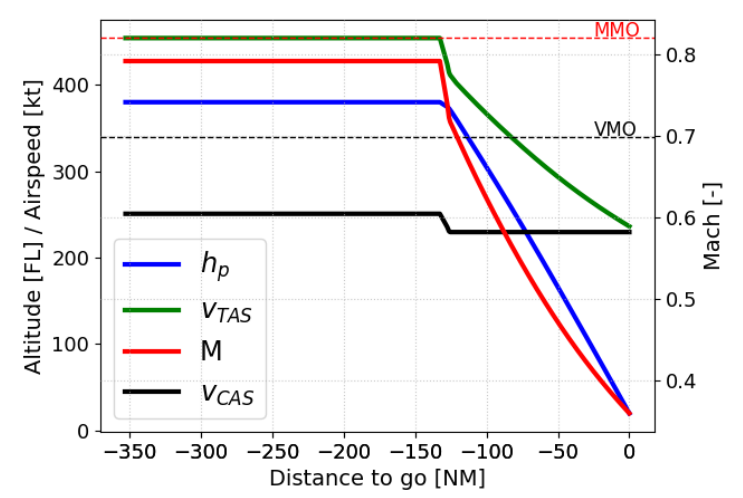

(b) Latest trajectory

Fig. 4. Earliest and latest trajectories
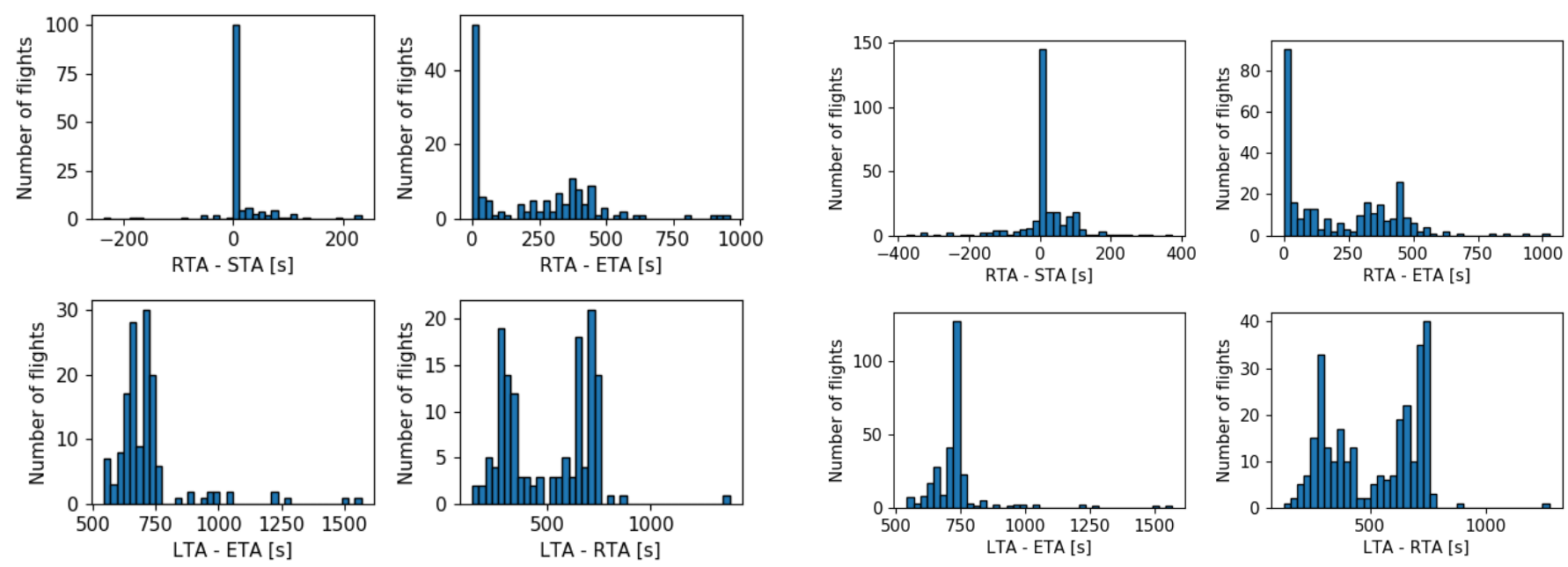

(a) Scenario 1
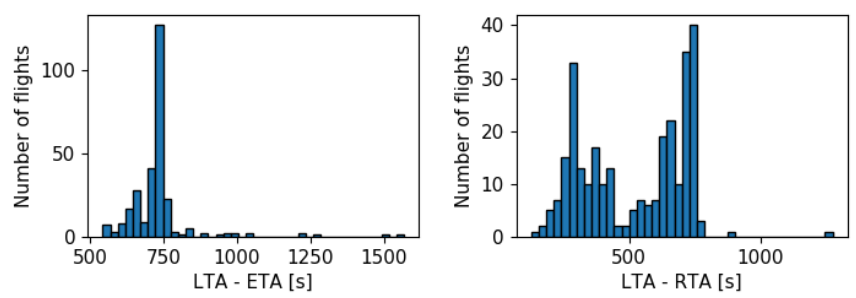

(b) Scenario 2

Fig. 5. Required time of arrival in the final approach fix

\section{RTA assignment results}

In Figs. 5(a) and 5(b), corresponding to scenario 1 and scenario 2, respectively, some interesting bar charts are shown regarding the RTA assignment in the FAF. For each subfigure, the top left panel represents the delay assigned to each flight, which is the difference between the RTA and the estimated time of arrival (STA). The bottom left panel shows the time window, which is the difference between the latest time of arrival (LTA) and the earliest time of arrival (ETA). Finally, the top and bottom right panels represent the difference of the RTA with the ETA and the LTA, respectively.

One of the most important parameters to be analyzed is the delay, which is to be minimized. Table II shows some statistical parameters of the assigned delay for both scenarios.

Regarding scenario $1,50 \%$ of the flights have no delay, and $75 \%$ of flights are below 26 seconds of delay. The worst delay is 234 seconds (less than 4 minutes), which is acceptable.

Regarding scenario 2, it can be observed that the results obtained are worse than those of scenario 1 . This is completely understandable as more traffic implies more difficulties in the RTA scheduling process: there is a higher frequency of aircraft entering the E-TMA, and higher delays have to be assigned in order to keep safe time separation between aircraft. Yet, the results are quite acceptable. There is an average delay of less than 1 minute, and $75 \%$ of the aircraft have less than 74

TABLE II

DELAY STATISTICAL PARAMETERS

\begin{tabular}{lcc}
\hline Statistical parameter & Scenario 1 & Scenario 2 \\
\hline Mean & $22.672 \mathrm{~s}$ & $44.885 \mathrm{~s}$ \\
Standard deviation & $48.31 \mathrm{~s}$ & $70.138 \mathrm{~s}$ \\
1st quartile & $0 \mathrm{~s}$ & $0 \mathrm{~s}$ \\
Median & $0.000174 \mathrm{~s}$ & $8.73 \mathrm{~s}$ \\
3rd quartile & $25.796 \mathrm{~s}$ & $74.31 \mathrm{~s}$ \\
Min & $0 \mathrm{~s}$ & $0 \mathrm{~s}$ \\
Max & $235.89 \mathrm{~s}$ & $374.09 \mathrm{~s}$ \\
\hline
\end{tabular}


seconds of delay. The worst delay is around 6 minutes.

Another interesting concept is the time window, which represents the interval of time in which an RTA can be assigned. The time window depends on the available routes, existing a different time window for each route. It is worth noting the fact that the feasible time windows for the different routes can overlap, as explained in [11]. This means that for a given RTA, there are many possible routes such that the RTA fits within the feasible time window. Therefore, separation can be achieved by tactically re-allocating the routes using closedloop instructions without modifying the enforced RTA.

The bottom left panels of Figs. 5(a) and 5(b) show only the total time window, which is computed with the LTA of the longest route and the ETA of the shortest route. The higher the time window, the higher the flexibility to assign an RTA to a given aircraft and the easier the scheduling process will be. The results are similar for both scenarios, as it is shown in Table III. time windows of at least 9 minutes are ensured for both scenarios, with $75 \%$ of the values above 10 minutes.

TABLE III

TIME WINDOW STATISTICAL PARAMETERS

\begin{tabular}{lcc}
\hline Statistical parameter & Scenario 1 & Scenario 2 \\
\hline Mean & $718.13 \mathrm{~s}$ & $729.67 \mathrm{~s}$ \\
Standard deviation & $153.31 \mathrm{~s}$ & $109.84 \mathrm{~s}$ \\
1st quartile & $646.46 \mathrm{~s}$ & $697.01 \mathrm{~s}$ \\
Median & $695.31 \mathrm{~s}$ & $730.86 \mathrm{~s}$ \\
3rd quartile & $729.35 \mathrm{~s}$ & $741.54 \mathrm{~s}$ \\
Min & $544.07 \mathrm{~s}$ & $544.07 \mathrm{~s}$ \\
Max & $1567.4 \mathrm{~s}$ & $1567.39 \mathrm{~s}$ \\
\hline
\end{tabular}

Finally, the top and bottom right panels are useful to determine where the RTA is assigned in the available time window. For both scenarios, there is a great amount of aircraft that are assigned an RTA equal to the ETA, which represent almost one third of the total arriving traffic. This means that a great amount of aircraft were flying with a high estimated CI value to land the earliest possible.

\section{Route assignment results}

As explained in section III-A4 there is a set of available routes for each aircraft that enable them to attain the assigned RTA at the FAF. Figure 6 shows the number of route assigned to the aircraft, being 5 the shortest route and 1 the longest one, corresponding to execute the complete tromboning procedure without shortcuts.

Most of the aircraft are assigned the shortest route (number 5), while few aircraft are assigned routes 4 and 3 and almost none routes 2 and 1 . This is due to the fact that the route assigner algorithm always tries to assign the shortest possible route, as long as the time separation is kept. Moreover, as it has been explained in Section III-C, almost one third of the aircraft have an RTA corresponding to the ETA, which enforces them to choose the shortest route.

Figure 7 shows the time separation between aircraft for both scenarios. For scenario 1, 29\% of flights have a time separation in the FAF of 120 seconds, while for scenario 2 this value increases to $53 \%$ of flights. As it has been aforementioned, the fact that there are more flights in scenario 2 makes more difficult the scheduling process, and less time separation can be ensured between the arriving aircraft.

\section{CONClusions And Future WORK}

This paper proposed to sequence traffic in the terminal maneuvering area (TMA) by means of 4D closed-loop instructions, enabling aircraft to fly continuous descent operations (CDO) trajectories from cruise level to the runway threshold. The assessment has been done in Berlin-Schönefeld airport, by using its trombone procedure. Results show that, after assigning required times of arrival (RTAs) and routes to every arriving aircraft, a time separation of at least 120 seconds is ensured in the final approach fix (FAF), while at least 90 seconds of time separation are ensured in the rest of waypoints of the trombone procedure.

With low traffic scenarios, like the baseline scenario presented in this paper, there are no problems in scheduling and merging the aircraft. However, when the traffic increases higher delays have to be assigned, and it is more difficult to keep a safe time separation. Moreover, it is important that the aircraft arrive to the TMA with enough separation in order to successfully schedule them during the arrival and approach phases.

Although the obtained results are very promising, there is still margin for improvement. First of all, the optimization model needs some changes. The next step would be to assign RTAs by taking into account not only the time window in the FAF, but also the time windows of all the other waypoints (including those of the STAR procedure), which will depend on the available routes of the tromboning. This way, the output of the optimization would be an RTA in the FAF, like before, and a route for each aircraft, removing the need for an external route assignment algorithm and ensuring a given time separation in all the waypoints of the arrival procedure.

In this paper, CDO trajectories have been generated with a semi-analytical method, in which some assumptions have to be made, such as for instance that no speed changes can be done during the cruise phase or that no altitude and speed constraints apply during the descent. In future work, this method will be enhanced in order to obtain more realistic results.

To sum up, it can be said that trombone procedures are a good solution to enable environmentally-friendly trajectories. They provide means to ensure comparable levels of capacity and safety to those obtained with traditional sequencing and merging techniques (i.e., open-loop vectoring), but with a higher level of efficiency. Furthermore, pilots do not loose situational awareness: they always know the remaining distance and route that they will follow until the runway threshold. However, further research is needed to assess the applicability of such technique, as the RTA and route assignment are not straight-forward tasks, and several factors like the complexity of the procedure or the traffic density could seriously degrade the separation between aircraft and thus, the safety of the operation. 


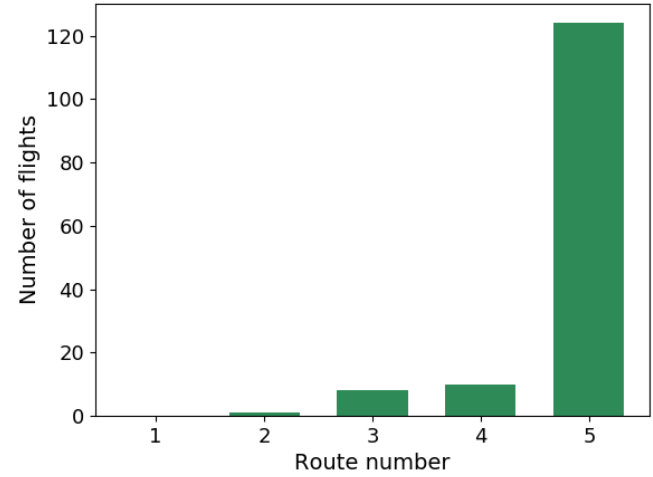

(a) Scenario 1

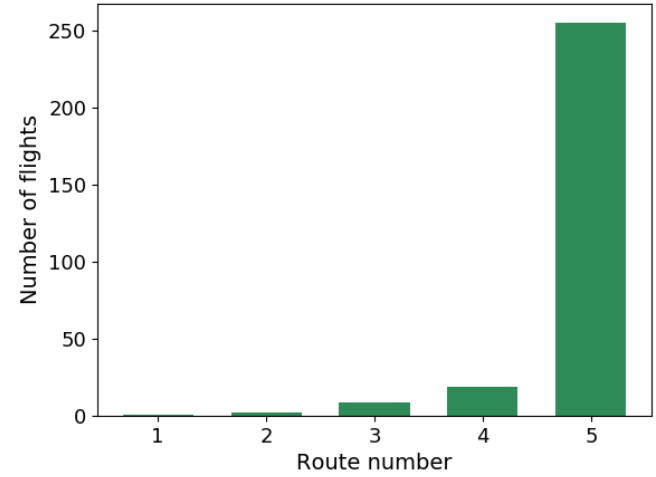

(b) Scenario 2

Fig. 6. Assigned routes

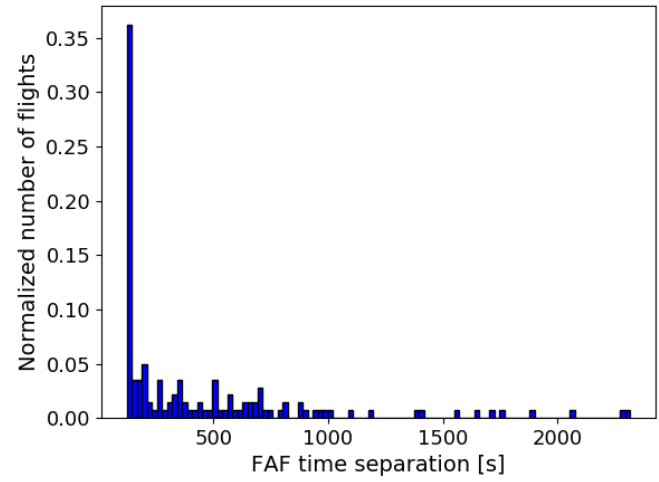

(a) Scenario 1

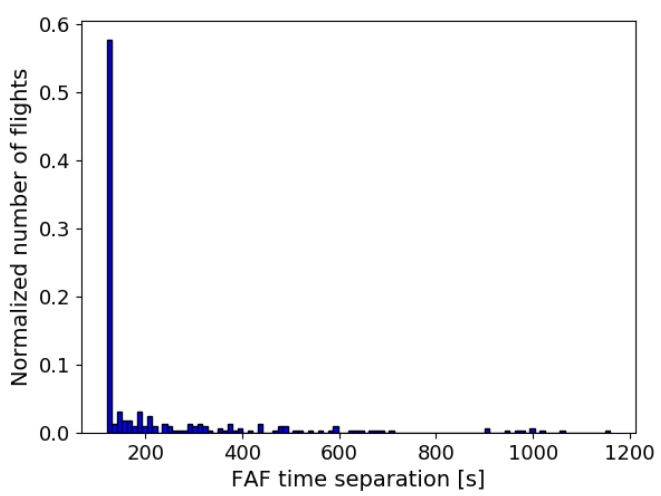

(b) Scenario 2

Fig. 7. Time separation in the final approach fix

\section{REFERENCES}

[1] L. Erkelens, "Research into new noise abatement procedures for the 21st century," in AIAA Guidance, Navigation, and Control Conference and Exhibit, ser. Guidance, Navigation, and Control and Co-located Conferences. Denver, CO: American Institute of Aeronautics and Astronautics, 2000.

[2] A. Warren and K. Tong, "Development of continuous descent approach concepts for noise abatement," in IEEE/AIAA 21st Digital Avionics Systems Conference (DASC), Irvine, CA, 2002.

[3] J. P. B. Clarke, N. T. Ho, L. Ren, J. A. Brown, K. R. Elmer, K. F. Zou, C. Hunting, D. L. McGregor, B. N. Shivashankara, K. Tong, A. W. Warren, and J. K. Wat, "Continuous descent approach: Design and flight test for Louisville international airport," Journal of Aircraft, vol. 41, no. 5, pp. 1054-1066, 2004.

[4] ICAO, "Continuous Descent Operations (CDO) Manual-Doc 9931/AN/476," (ICAO), International Civil Aviation Organization, Montreal, Quebec, Canada, Tech. Rep., 2010.

[5] H. Fricke, C. Seiß, and R. Herrmann, "Fuel and Energy Benchmark Analysis of Continuous Descent Operations," in USA/Europe Air Traffic Management Research and Development Seminar, vol. 23, no. ATC Quarterly Special Issue, Washington D.C., USA, 2015, pp. 83-108.

[6] SESAR Joint Undertaking, "European ATM Master Plan. The roadmap for delivering high performing aviation for Europe," Brussels, Tech. Rep., 2015.

[7] J. K. Klooster, A. D. Amo, and P. Manzi, "Controlled Time-of-Arrival Flight Trials," in 8th USA/Europe air traffic management research and development seminar, Napa, CA, 2009.
[8] P. M. A. de Jong, F. J. L. Bussink, R. Verhoeven, N. de Gelder, M. M. V. Paassen, and M. Mulder, "Time and Energy Management during Descent and Approach: a human-in-the-loop study," Journal of Aircraft, vol. 54, no. 1, pp. 177-189, 2017.

[9] X. Prats, B. Bendris, R. Dalmau, J. Montolio, B. Day, H. Lenz, and R. Kohrs, "4D Continuous Descent Operations Supported by an Electronic Flight Bag* A Human-in-the-loop Study," in IEEE/AIAA 35th Digital Avionics Systems Conference (DASC), Sacramento, CA, 2016.

[10] A. Pawelek, R. Dalmau, P. Lichota, and X. Prats, "Arrival traffic synchronisation with Required Time of Arrivals for fuel-efficient trajectories," in 17th AIAA Aviation Technology, Integration, and Operations Conference (ATIO), Denver, CO, 2017.

[11] R. Dalmau, J. Alenka, and X. Prats, "Combining the assignment of pre-defined routes and RTAs to sequence and merge arrival traffic," in 17th AIAA Aviation Technology, Integration, and Operations Conference (ATIO), Denver, CO, 2017.

[12] L. Boursier, B. Favennec, E. Hoffman, A. Trzmiel, F. Vergne, and K. Zeghal, "Merging Arrival Flows Without Heading Instructions," in USA/Europe Air Traffic Management Research and Development Seminar, no. July, 2007, pp. 1-8.

[13] D. Ivanescu, C. Shaw, C. Tamvaclis, and T. Kettunen, "Models of Air Traffic Merging Techniques: Evaluating Performance of Point Merge," in 9th AIAA Aviation Technology, Integration, and Operations (ATIO) Conference, Hilton Head, South Carolina, September, 2009.

[14] A. E. Bryson and Y.-C. Ho, Applied optimal control : optimization, estimation, and control. New York, USA: Taylor and Francis Group, 1975.

[15] C. de Boor, "On calculating with B-splines," Journal of Approximation 
Theory, vol. 6, no. 1, pp. 50-62, 1972.

[16] P. M. A. de Jong, J. J. van der Laan, A. C. Veld, M. M. van Paassen, and M. Mulder, "Wind-Profile Estimation Using Airborne Sensors," Journal of Aircraft, vol. 51, no. 6, pp. 1852-1863, 2014.

[17] S. G. Park, P. Dutta, and P. K. Menon, "Optimal Trajectory Option Sets for In-Flight Climb-Descend Trajectory Negotiations," in 17th AIAA Aviation Technology, Integration, and Operations Conference (ATIO). Denver, CO: AIAA, June 2017.

[18] D. Poles, A. Nuic, and V. Mouillet, "Advanced aircraft performance modelling for ATM: Analysis of BADA model capabilities," in 29th Digital Avionics Systems Conference. Brétigny-sur-Orge (France): EUROCONTROL, 2010.

[19] C. Guéret, C. Prins, and M. Sevaux, Applications of optimization with Xpress-MP, Eyrolles, Ed. Paris, France: Dash Optimization Ltd., 2007.

[20] B. A. McCarl and T. H. Spreen, Applied mathematical programming using algebraic systems. FL: Texas A\&M University and University of Florida, 2011.

[21] Eurocontrol, DDR2 Reference Manual for General Users 2.9.4, Sept. 2017.

[22] E. Gallo, "Method for optimum economy cruise speed in an aircraft," U.S. Patent US 2016/0 260333 A1, Sep 8, 2016. 